\title{
Correction to: morphometric characterization and monitoring of Al- Asfar Lake in Al-Hasa governorate, Saudi Arabia
}

\author{
Noor Alhajji ${ }^{1} \cdot$ Ali Elbattay $^{2} \cdot$ Fahad Almutlaq $^{1}$
}

Published online: 23 November 2021

C) Saudi Society for Geosciences 2021

Correction to: Arabian Journal of Geosciences (2021) 14:1109

https://doi.org/10.1007/s12517-021-07393-9

In this article the affiliation details of authors "Ali Elbattay and Fahad Almutlaq" were incorrectly given as "Geography Department, King Saud University, Riyadh, Saudi Arabia" and "The International Center for Biosaline Agriculture (ICBA), Al Ruwayyah 2, Academic City, Dubai, United Arab Emirates" but should have been "The International Center for Biosaline Agriculture (ICBA), Al Ruwayyah 2, Academic City, Dubai, United Arab Emirates" and "Geography Department, King Saud University, Riyadh, Saudi Arabia".

The original article has been corrected.

The online version of the original article can be found at https:// doi.org/10.1007/s12517-021-07393-9

Fahad Almutlaq

Falmutlaq@ksu.edu.sa

1 Geography Department, King Saud University, Riyadh,

Saudi Arabia

2 The International Center for Biosaline Agriculture (ICBA), Al Ruwayyah 2, Academic City, Dubai, United Arab Emirates 